\title{
Clipping through a mirror: First reported successful MitraClip in a patient with dextrocardia
}

\author{
Antonio H. Frangieh, Carolin Sonne, Philipp Moritz Rumpf, \\ Albert Markus Kasel, Ilka Ott \\ Department of Cardiology, Deutsches Herzzentrum München, \\ Technische Universität München, Munich, Germany
}

Reported herein is a 69-year-old male patient known to have dextrocardia. He suffers from triple vessel disease with severe ischemic heart failure (left ventricular ejection fraction of $18 \%$ ), with history of myocardial infarction 32 years ago, coronary artery bypass graft 31 years ago and numerous percutaneous coronary interventions on the bypass grafts and native coronaries. The patient presented with conduction disturbances and symptomatic ventricular tachycardia (VT), and had a pacemaker and an implantable cardioverter defibrillator 5 years prior to admission, followed by endocardial VT ablation for recurrent symptomatic VTs. The patient was admitted to the documented center for dyspnea New York Heart Association class III under optimal medical treatment and was diagnosed with a severe mitral regurgitation (MR) grade III/IV due to mixed mitral valve disease (functional and degenerative) with posterior mitral leaflet retraction and anterior mitral leaflet pseudo-prolapse at the level of segment 2 (Fig. 1A). The heart team decided for percutaneous clipping using the MitraClip ${ }^{\circledR}$ system (Abbott Vascular, Santa Clara, CA, USA) because of suitable anatomy and high-risk of re-thoracotomy (logistic Euroscore 19.5\%). He underwent MitraClip implantation under general anesthesia and transesophageal echocardiography. Echo-guided transseptal puncture was performed without difficulty, besides that the transseptal needle was facing towards the anatomical left atrium in a left to right direction on fluoroscopy (Fig. 1B). To achieve sufficient navigation the MitraClip was inserted $90^{\circ}$ counterclockwise into the Clip Delivery System handle. Throughout the procedure the steering was difficult and moving the system in any direction was quite unususal. After two trials, grasping the middle part of the second segment with good leaflet insertion was possible (Supplementary Movie - see journal website) and MR reduction was achieved (Fig. 1C). The placement of a second clip was not performed because of high transmitral gradient (dPmean: $5 \mathrm{~mm} \mathrm{Hg}$, PHT $137 \mathrm{~ms}$ and MVA $1.61 \mathrm{~cm}^{2}$ ).

After the release of the MitraClip, the echocardiography measurements showed the clip in stable position in situ, residual MR grade II with two residual jets from either side of the clip and a good valve opening (dPmean: $4 \mathrm{~mm} \mathrm{Hg}$ ). Transseptal procedure time was $90 \mathrm{~min}$ and was higher than routine MitraClip procedures due to steering and imaging difficulties. The most challenging part for the operator was the steering of the Clip and for the echocardiographer orientation of the probe and generation of adequate views without standard views. It should be stressed that in these cases good communication between the operator and echocardiographer is essential for success of the procedure. After the transseptal puncture the guiding was done carefully and conscientiously. The common steering movements were not suited for guidance due to the altered anatomy. Thus, the first step of the operator was to explore the steering behavior by the use of live three-dimensional-echoimaging. Afterwards the commands for guiding and steering were determined either technically by the operator or anatomically by the echocardiographer.

Chest X-ray after the procedure showed the clip in place "on the right side of the chest" (Fig. 1D).

Address for correspondence: Dr. Ilka Ott, Deutsches Herzzentrum München, Lazarettstrasse 36, 80636 München, Germany, tel: +49-89-1218-0, e-mail: ott@dhm.mhn.de

Received: 07.05.2017 Accepted: 25.07.2017 


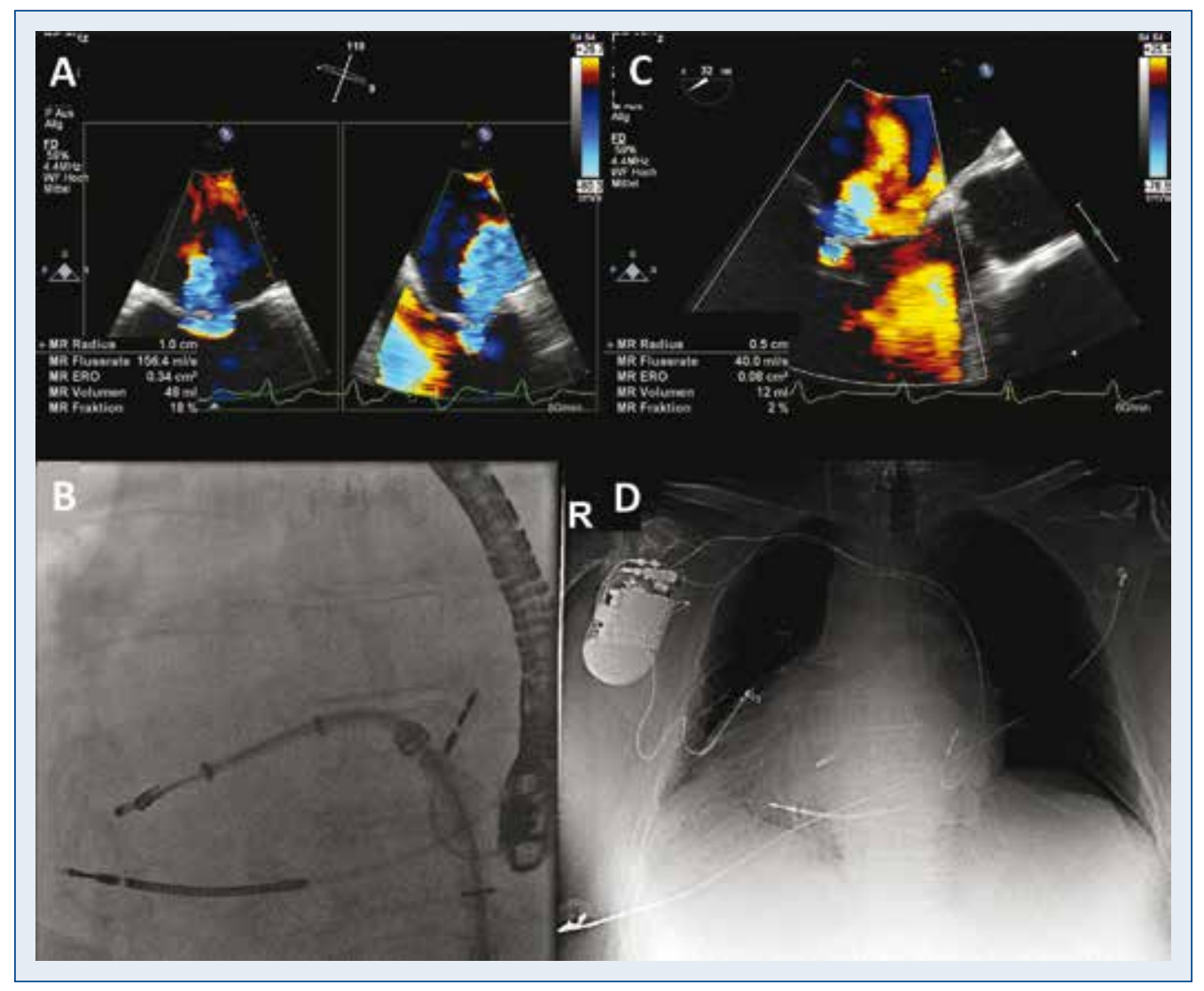

Figure 1. Echocardiography shows severe mitral valve regurgitation before $(\mathbf{A})$ and moderate mitral valve regurgitation after MitraClip implantation (C). Fluoroscopy shows the position of the MitraClip as in a mirror (B); D. Chest-X-ray after the procedure.

The patient was discharged 3 days after intervention. At 1-year follow-up, the control echocardiography showed a stable functional result and the patient experienced pronounced clinical improvement.

This is, according to available research, the first published case of a successful MitraClip in a patient with situs inversus for treatment of severe MR. A previously reported case with dextrocardia was complicated with delayed clip detachment after intervention and death of the patient [1]. Unusual anatomy can make the MitraClip procedure chal- lenging, since the system guidance and steerability becomes more difficult, and imaging guidance is unpredictable.

Conflict of interest: None declared

\section{Reference}

1. Nielsen NE, Baranowski J, Boano G, Nylander E, Wallby L. MitraClip in a Patient with Dextrocardia and Scoliosis Complicated by Delayed Clip Detachment. In: Atlas of Percutaneous Edge-to-Edge Mitral Valve Repair, Springer 2012: 515-517. 